\title{
Modeling Fluid Flow Effects in Close Binary and Protoplanetary Systems
}

\author{
M. M. Montgomery \\ Physics Department, University of Central Florida, PY 132, Orlando, Florida, 32816, USA \\ email: montgomery@physics .ucf .edu
}

\begin{abstract}
Accretion disks around some white dwarfs in Cataclysmic Variables are thought to tilt around the line of nodes by the lift force acting at the disk's center of pressure. We investigate whether protoplanetary disks can also experience disk tilt. We find that lift may be possible by an asymmetric, net uni-directional, in-falling gas/dust stream overflowing a bluff body (e.g., Class I sources) or inner annuli of young Class II sources if gas/dust is still in-falling and the aspect ratio and disk surface area are large enough. However, inner disks of Class II sources LkCa 15, UX Tau A, and Rox 44 are not large enough, and therefore disk tilt is not likely.
\end{abstract}

Keywords. accretion, accretion disks; hydrodynamics; planetary systems: protoplanetary disks; novae, cataclysmic variables

\section{Introduction}

Montgomery \& Martin (2010) find that an asymmetric gas stream flowing over a disk rim in a Cataclysmic Variable system can result in a lift force acting at the disk's center of pressure, resulting in a disk tilt around the line of nodes. Gravitational forces cause the tilted disk to precess retrogradely, and the source of precession is tidal torques like those by the Moon and Sun on the oblate, spinning, tilted Earth (Montgomery 2009). In this work, we apply this model to Class I and Class II sources to find if these disks can also tilt by the lift force. We use $04108+2803 \mathrm{~B}$ as our model Class I source and LkCa 15, UX Tau A, and Rox 44 as our model pre-transitional disk Class II sources. We consider in-falling gas/dust particles that turn and flow over/under the disk and mass transfer effects through the disk, but we do not consider effects due to jets and winds.

\section{Lift in Protostellar Disks}

Observational Data. In Tables 1 \& 2, we list the stellar and disk property values assumed in Chiang \& Goldreich (1999) and (Espaillat et al. 2010) for the Class I source 04108+2803B and for Class II pre-transitional disk sources LkCa 15, UX Tau A, Rox 44 that may have young planets forming, respectively. In these tables, the target star mass $M$, stellar radii $R$, mass transfer rate $\dot{M}$ through the disk, disk inner $(i)$ and outer (o) radius $r$, and disk height $z$ are given. The inner wall radius $\left(r_{\text {wall }}^{i}\right)$ and the inner disk radius $\left(r_{\text {disk }}^{i}\right)$ differ in values, since the inner wall location is based upon the dust sublimation radius whereas the inner disk location is a constrained value (see Espaillat et al. 2010). As Class I sources have no gap, the inner disk rim and disk mass are assigned $r_{w a l l}^{i}$ and $M_{\text {disk }}^{o}$, respectively. The rim disk height $z_{\text {wall }}^{o}$ is taken to be four times the gas scale height, a conservative value, as determined by Equation (3f) of Chiang \& Goldreich (1999). The dashed lines indicate no known data and N/A means Not Applicable. Note that the inner disk is geometrically thin relative to the gap width and the outer disk height. 
Table 1. Class I and Class II Stellar Property Values

Class

\begin{tabular}{llcccl} 
Source & Target & Spectral Type & $M_{1}\left(\mathrm{M}_{\odot}\right)$ & $R_{1}\left(\mathrm{R}_{\odot}\right)$ & $\dot{M}\left(\mathrm{M}_{\odot} \mathrm{yr}^{-1}\right)$ \\
\hline I & 04108+2803B & - & 0.5 & 2.5 & - \\
II & LkCa 15 & K3 & 1.3 & 1.6 & $0.33 \times 10^{-8}$ \\
II & UX Tau A & G8 & 1.5 & 1.8 & $1.1 \times 10^{-8}$ \\
II & Rox 44 & K3 & 1.3 & 1.6 & $0.93 \times 10^{-8}$
\end{tabular}

Table 2. Pre-transitional disc Candidates \& Assumed disc Property Values

\begin{tabular}{llcccccccc}
$\begin{array}{l}\text { Class } \\
\text { Source }\end{array}$ & Target & $\begin{array}{rlcccc}r_{\text {wall }}^{i} \\
(\mathrm{AU})\end{array}$ & $\begin{array}{c}z_{\text {wall }}^{i} \\
(\mathrm{AU})\end{array}$ & $\begin{array}{c}r_{\text {disk }}^{i} \\
(\mathrm{AU})\end{array}$ & $\begin{array}{c}M_{\text {disk }}^{i} \\
\left(M_{\odot}\right)\end{array}$ & $\begin{array}{c}r_{\text {wall }}^{o} \\
(\mathrm{AU})\end{array}$ & $\begin{array}{c}z_{\text {wall }}^{o} \\
(\mathrm{AU})\end{array}$ & $\begin{array}{c}M_{\text {disk }}^{o} \\
\left(M_{\odot}\right)\end{array}$ & $\begin{array}{c}r_{\text {disk }}^{o} \\
(\mathrm{AU})\end{array}$ \\
\hline I & 04108+2803B & 0.07 & - & $\mathrm{N} / \mathrm{A}$ & $\mathrm{N} / \mathrm{A}$ & $\mathrm{N} / \mathrm{A}$ & 268 & $1.5 \times 10^{-2}$ & 270 \\
II & LkCa 15 & 0.15 & 0.017 & $<0.19$ & $<2 \times 10^{-4}$ & 58 & 12.9 & $10 \times 10^{-2}$ & 300 \\
II & UX Tau A & 0.15 & 0.009 & $<0.21$ & $<0.6 \times 10^{-4}$ & 71 & 13.8 & $4 \times 10^{-2}$ & 300 \\
II & Rox 44 & 0.25 & 0.034 & $<0.4$ & $<0.8 \times 10^{-4}$ & 36 & 9.9 & $3 \times 10^{-2}$ & 300 \\
\hline
\end{tabular}

Analytical Model. Unequal in-falling gas/dust parcels on different flow paths above and below the disk can result in different pressures on the disk faces and thus a disk tilt. Equating Equations (4) and (7) in Montgomery \& Martin (2010), disk tilt results if

$$
\frac{A_{s}}{2} \rho\left(v_{d i s k}^{o}\right)^{2}\left(1-\beta^{2}\right)>\frac{8 G \Sigma m M_{1} \sin \theta}{9 r_{d}^{2}}+\frac{3 r_{d} G m M_{2} \sin \theta}{8\left(d^{2}+\frac{9}{16} r_{d}^{2}-\frac{3}{2} r_{d} d \cos \theta\right)^{3 / 2}} \text {. }
$$

In this relation, $A_{s}$ is disk face surface area, $\rho=10^{-5} \mathrm{~kg} \mathrm{~m}^{-3}$ is in-falling gas/dust density (Larson, 1969), $\beta=0.9$ is fraction of the gas velocity flowing under to over the disk, $G$ is the universal gravitational constant, $\Sigma m$ is disk mass, $m \approx M_{\text {disk }}^{o} / 100,000$ is mass of gas/dust parcel, $r_{d}$ is radius of a circular and geometrically thin disk, $M_{2}$ is mass of forming planet in disk, $d$ is separation distance, and $\theta=4^{\circ}$ is minimum disk tilt angle.

Results - Class I Sources. From conservation of energy, $\left|v_{d i s c}^{o}\right|$ is found by assuming a gas/dust parcel falls from rest at infinity to the disk rim. Upon substitution of $A_{s}=$ $\pi\left(r_{\text {disc }}^{o}\right)^{2}$ and $M_{2} \ll M_{1}$, the left hand side of Equation (2.1) is greater than the right hand side and thus, disk tilt seems likely.

Results - Young Class II Sources. In young Class II sources, $M_{2} \ll M_{1}$ since no gap has formed. For hypothetical younger versions of LkCa 15, UX Tau A, and Rox 44, we find in-fall speeds to the disk rim are subsonic and disk surface areas are large and, therefore, a tilt on outer disk annuli is not likely. However, as the gas/dust parcels drop further into the potential well, speeds increase and a net asymmetric unidirectional gas/dust stream flowing over and under the inner annuli of the disk may result in disk tilt.

Results - Class II Sources. In Equation (2.1), we replace $r_{d i s c}^{o}$ with $\left(r_{d i s c}^{o}-r_{\text {wall }}^{o}\right)$ and allow $M_{2} \ll M_{1}$. For LkCa 15, UX Tau A, and Rox 44, inner disks do not have enough surface area and, therefore, disk tilt will not occur.

\section{Acknowledgement}

The author is grateful for financial support from the IAU to attend this symposium.

\section{References}

Chiang, E. L. \& Goldreich, P. 1999, ApJ, 519, 279

Espaillat, C., D’Alessio, P., Hernández, J., Nagel, E., Luhman, K. L., Watson, D. M., Calvet, N., Muzerolle, J., \& McClure, M. 2010, ApJ, 717, 441

Larson, R. B. 1969, MNRAS, 145, 271

Montgomery, M. M. 2009, ApJ, 705, 603

Montgomery, M. M. \& Martin, E. L. 2010, ApJ, 722, 989 